\title{
Theoretical Study of the $\mathrm{BaTiO}_{3}$ Powder's Volume Ratio's Influence on the Output of Composite Piezoelectric Nanogenerator
}

\author{
Xi Zhou ${ }^{1}$, Qi Xu ${ }^{2}$, Suo Bai ${ }^{2}$, Yong Qin ${ }^{2,3, *}$ and Weisheng Liu ${ }^{1, *}$ \\ 1 Key Laboratory of Nonferrous Metals Chemistry and Resources Utilization of Gansu Province, State Key \\ Laboratory of Applied Organic Chemistry, College of Chemistry and Chemical Engineering, Lanzhou \\ University, Lanzhou 730000, China; zhoux13@lzu.edu.cn \\ 2 Institute of Nanoscience and Nanotechnology, School of Physical Science and Technology, Lanzhou \\ University, Lanzhou 730000, China; xuq10@lzu.edu.cn (Q.X.); baisuo@lzu.edu.cn (S.B.) \\ 3 School of Advanced Materials and Nanotechnology, Xidian University, Xi'an 710071, China \\ * Correspondence: qinyong@lzu.edu.cn (Y.Q.); liuws@lzu.edu.cn (W.L.); \\ Tel.: +86-931-891-5038 (Y.Q.); +86-931-891-2576 (W.L.)
}

Received: 6 April 2017; Accepted: 5 June 2017; Published: 9 June 2017

\begin{abstract}
The combination of the piezoelectric materials and polymer is an effective way to make the piezoelectric nanogenerator (PENG) possess both the polymer's good flexibility and ferroelectric material's high piezoelectric coefficient. The volume ratio of ferroelectric material in the composite is an important factor that determines the PENG's output performance. In this paper, the $\mathrm{BaTiO}_{3} /$ polydimethylsiloxane (PDMS) composite PENG was demonstrated as having an optimal volume ratio (46\%) at which the PENG can output its highest voltage, and this phenomenon can be ascribed to the trade-off between the composite PENG's top electrode charge and its capacitance. These results are of practical importance for the composite PENG's performance optimization.
\end{abstract}

Keywords: piezoelectric nanogenerator; composite material; optimal volume ratio; performance optimization

\section{Introduction}

With the development of nanotechnology and micro-electromechanical systems (MEMS), the implantable medical devices, personal electronics, and distributed networks have been widely explored to facilitate people's daily life. Despite the convenience brought by these technologies, some potential problems begin to emerge because of the limited capacity of traditional batteries used in the above devices. It is especially inconvenient to replace depleted batteries for implantable medical devices because it is a miserable experience for patients and a high risk surgical procedure for surgeons. Even worse, among the numerous nodes in distributed sensor networks, it is almost impossible to find the nodes running out of power. To solve the above-mentioned problems, piezoelectric nanogenerator [1], which can harvest abundant in vivo, in vitro body motion energy and environmental mechanical energy, is developed to provide power supply for the implantable medical devices [2-7], personal electronics [8-15], and distributed sensor networks. As a power supply, the electric output and endurance are two key issues for piezoelectric nanogenerator's (PENG's) practical applications. Composite PENG, based on inorganic piezoelectric material and organic polymer, is a promising candidate self-powered generators with both good flexibility and high piezoelectric coefficient.

For the preparation of composite PENG, the key issues to be solved are how to disperse the ferroelectric materials uniformly in the polymer matrix and to find out at which ratio of the ferroelectric material, the PENG can output the maximum electricity. Due to the polymer matrix's sticky and 
hydrophobic properties, it is hard to obtain a well-distributed composite by dispersing the ferroelectric material directly into the polymer matrix. In order to solve these problems, multiwalled carbon nanotubes [16,17], Cu nanorods [18], ZnS nanorods [19], virus templates [20], and bacterial cellulose [21] fillers have been used to facilitate the dispersion of the ferroelectric material, because the ferroelectric material can form a complex mixture with filler-based-entangled network structure. Some of the research results showed the existence of an optimal ratio in the ferroelectric material's ratio in the composite PENG [18,21,22]. Therefore, systematic theoretical research is needed to study this phenomenon for the development of composite PENG.

In this paper, we studied the output voltage of the $\mathrm{BaTiO}_{3} / \mathrm{PDMS}$ composite PENG with different volume ratios of evenly distributed $\mathrm{BaTiO}_{3}$ cubes, and found the existence of an optimal volume ratio $(46 \%)$ of the $\mathrm{BaTiO}_{3}$ cubes at which the PENG can output the highest voltage, resulting from the trade-off between the surface charge and capacitance of the composite PENG.

\section{Methods and Structure}

To obtain the composite PENG's open circuit voltage, we need to solve the following governing equations for piezoelectric materials:

$$
\begin{gathered}
\sigma_{\mathrm{p}}=c_{\mathrm{pq}} \varepsilon_{\mathrm{q}}-e_{\mathrm{kp}} E_{\mathrm{k}} \\
D_{i}=e_{\mathrm{iq}} \varepsilon_{\mathrm{q}}+\kappa_{i \mathrm{k}} E_{\mathrm{k}}
\end{gathered}
$$

where $\sigma_{\mathrm{p}}$ is the stress tensor, and $\varepsilon_{\mathrm{q}}$ is the strain tensor. To keep the tensor equation compact, the Voigt notion was used to reduce the $3 \times 3$ symmetric stress tensor $\sigma_{\mathrm{mn}}$ and strain tensor $\varepsilon_{\mathrm{mn}}$ where $m, n \in(x, y, z)$, to 6-dimensional vectors $\sigma_{\mathrm{q}}$ and $\varepsilon_{\mathrm{p}}$ where $q, p \in(x x, y y, z z, y z, z x, x y), c_{\mathrm{pq}}$ is the linear elastic constant, $e_{\mathrm{kp}}$ is the linear piezoelectric coefficient, $\kappa_{i \mathrm{k}}$ is the dielectric constant, $E_{\mathrm{k}}$ is the electric field, and $D_{i}$ is the electric displacement. In this study, these equations are solved by the COMSOL software package (5.1, COMSOL Co., Ltd., Shanghai, China).

The structure of the simulated $\mathrm{BaTiO}_{3}$ /PDMS composite PENG is schematically shown in Figure 1, where the $\mathrm{BaTiO}_{3} / \mathrm{PDMS}$ composite is sandwiched between the top and bottom electrodes. The size of the PDMS matrix is $460 \times 460 \times 40 \mu \mathrm{m}$, and the size of the individual $\mathrm{BaTiO}_{3}$ cube poling along the $z$ axis is $30 \times 30 \times 30 \mu \mathrm{m}$, and the cubes are uniformly distributed in the matrix. In practice, it is inevitable for the $\mathrm{BaTiO}_{3}$ particles/polymer based PENG to have polymer between the electrode and $\mathrm{BaTiO}_{3}$. As shown in Figure 1, PDMS layer between the electrode and the $\mathrm{BaTiO}_{3}$ cubes in $5 \mu \mathrm{m}$ thickness was used to mimic this situation. In practical calculations, the electrodes are not added, the bottom of the composite was ground and fixed, the charge on the side walls and top is set to be zero, a stress of $0.5 \mathrm{MPa}$ is exerted on the top surface. $\mathrm{BaTiO}_{3}$ /PDMS composite containing, 9, 16, 25, 36, 49, 64, 81, 100, 121, 144, 169,196 , and $225 \mathrm{BaTiO}_{3}$ cubes are respectively calculated. The corresponding volume ratios are $2.9 \%$, $5.1 \%, 8.0 \%, 11.5 \%, 15.6 \%, 20.4 \%, 25.8 \%, 31.9 \%, 38.6 \%, 45.9 \%, 53.9 \%, 62.5 \%$, and $71.8 \%$, respectively. After the calculation, the average electric potential of the top surface is the PENG's open circuit voltage.

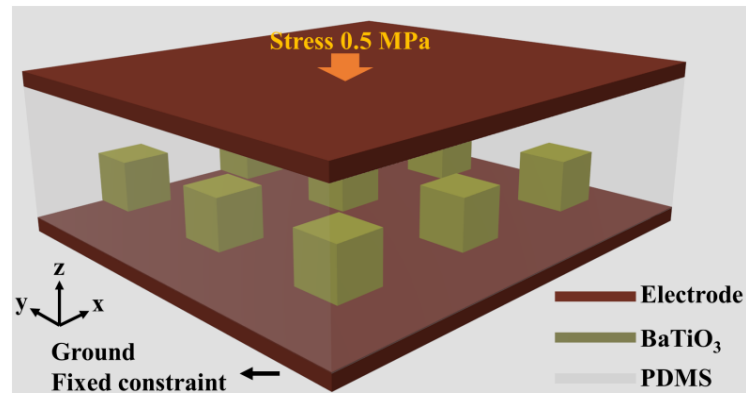

Figure 1. Schematic illustration of the $\mathrm{BaTiO}_{3} /$ polydimethylsiloxane (PDMS) composite piezoelectric nanogenerator (PENG). 


\section{Results and Discussion}

As shown in Figure 2a, the open circuit voltage of $\mathrm{BaTiO}_{3} / \mathrm{PDMS}$ composites with different volume ratios of $\mathrm{BaTiO}_{3}$ cubes are calculated. At the volume ratio of $45.9 \%$, the composite PENG reached its max voltage of $1.275 \mathrm{~V}$. To interpret this phenomenon, the composite PENG is approximated as a capacitor whose charge is generated by piezoelectric effects. Below this approximation, the voltage of the PENG can be calculated.

$$
V=Q / C
$$

where, $Q$ is the electric charge and $C$ is the composite PENG's capacitance. The accuracy of this approximation is examined by comparing the voltage calculated by $Q / C$ with that obtained directly by solving the coupled piezoelectric governing equation through finite element method (FEM). As shown in Figure $2 b$, we can see this approximation has a rather high accuracy when the $\mathrm{BaTiO}_{3}$ cubes' ratio is larger than $31.9 \%$. Below this volume ratio, the voltage obtained by $Q / C$ is higher than that obtained by solving the coupled piezoelectric governing equation. This discrepancy is due to the fact that the external forces on the deformation of PENG are not considered. When the volume ratio of $\mathrm{BaTiO}_{3}$ cubes is $31.9 \%$, the composite is stiff and the PENG's deformation under external force is small, as shown in Figure 2c. In contrast, when the volume ratio of the $\mathrm{BaTiO}_{3}$ cubes in the composite is low, under the external force, the deformation of the PDMS filling between the $\mathrm{BaTiO}_{3}$ cubes is large as shown in Figure 2d. The capacitance of the plate capacitor is

$$
C=\varepsilon_{0} \kappa S / d
$$

where, $\varepsilon_{0}$ is the vacuum permittivity, and $\mathcal{\kappa}$ is the composite's permittivity. The calculated capacitance of the PENG with low $\mathrm{BaTiO}_{3}$ cubes is smaller than the actual value without consideration of the deformation of the PENG under external force. Therefore, the voltage calculated on the basis of this capacitance is larger than the actual value. With the increase of $\mathrm{BaTiO}_{3}$ cubes' volume ratio, the composite becomes stiffer and stiffer, so the PENG's deformation can be neglected and the voltages calculated by the above two methods is consistent, as shown in Figure $2 \mathrm{~b}$. As the optimal volume ratio locates at $45.9 \%$, it is sufficient to interpret this phenomenon by comparing the volume ratio ranging from $31.9 \%$ to $71.8 \%$. In this volume ratio range, the Equation (3) has a rather good accuracy in describing the PENG's voltage.

Results of the surface charge and the capacitance of the PENG are shown in Figure 3a. With the increase of $\mathrm{BaTiO}_{3}$ cube's ratio, the value of PENG's capacitance increases gradually and toward a saturated value, because the permittivity of $\mathrm{BaTiO}_{3}$ is much higher than that of the PDMS. In contrast, the surface charge first increases with the $\mathrm{BaTiO}_{3}$ 's ratio and then decreases when the $\mathrm{BaTiO}_{3}$ 's ratio is further increased, because the surface charge of the composite PENG is induced by the charge generated by the $\mathrm{BaTiO}_{3}$ cubes under external force and the electric charge in the ferroelectric materials composed of two parts [23]. One is the surface charge caused by the discontinuous polarization across the ferroelectric material's surface and this value is proportional to the surface stress exerted on the ferroelectric material. The other one is the body charge caused by the gradient of the polarization, and this value is proportional to the gradient of the stress field in the ferroelectric material. The calculated average pressure and total force on the $\mathrm{BaTiO}_{3}$ cube's top surface are shown in Figure 3a. When the $\mathrm{BaTiO}_{3}$ 's ratio is increased, the average stress applied on the $\mathrm{BaTiO}_{3}$ cubes decreases, because the $\mathrm{BaTiO}_{3}$ cube in the polymer matrix has the property to concentrate the stress on it. As the cubes increase, the stress is more dispersed. On the one hand, the total force applied on the $\mathrm{BaTiO}_{3}$ cubes reaches its max at the volume ratio of $62.5 \%$. On the other hand, as the $\mathrm{BaTiO}_{3}$ cubes' ratio increases, the stress gradient in $\mathrm{BaTiO}_{3}$ cubes decreases. So the charge of the top has a maximum within this volume ratio range. With the trade-off of the capacitance and top electrode charge, the composite PENG reached its maximum output, when the $\mathrm{BaTiO}_{3}$ ratio is $45.9 \%$. 
a

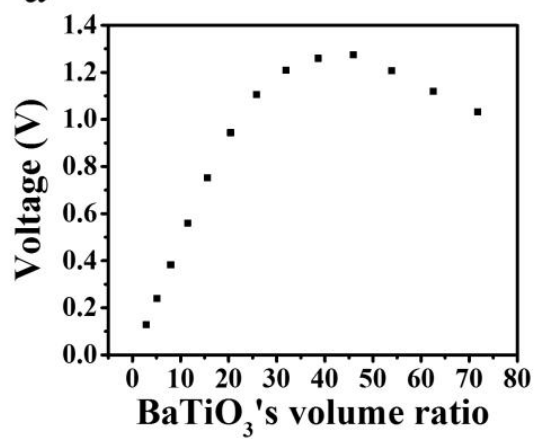

b

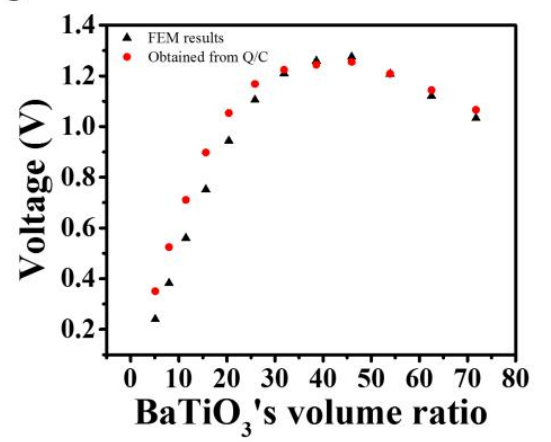

d

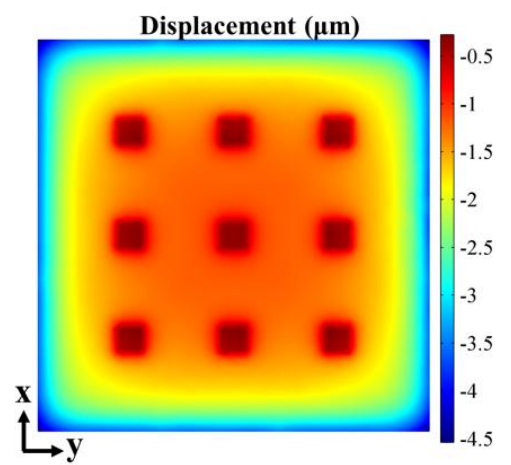

Figure 2. (a) The open circuit voltage of the composite PENG containing different volume ratios of $\mathrm{BaTiO}_{3}$ cubes; (b) The comparison of the voltage obtained $Q / C$ and that directly calculated from the coupled piezoelectric governing equation. The distribution of stress on the $\mathrm{BaTiO}_{3}$ cubes' top surface with volume ratio of $31.9 \%$ (c) and $2.9 \%$ (d).

a

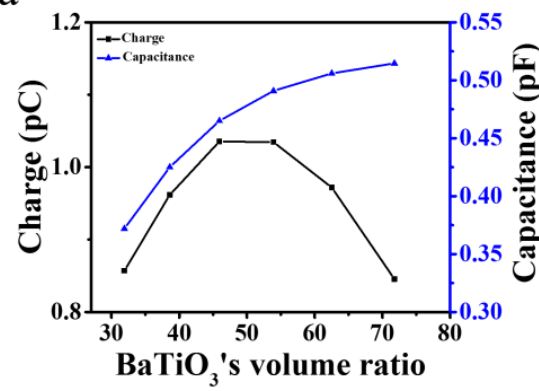

C

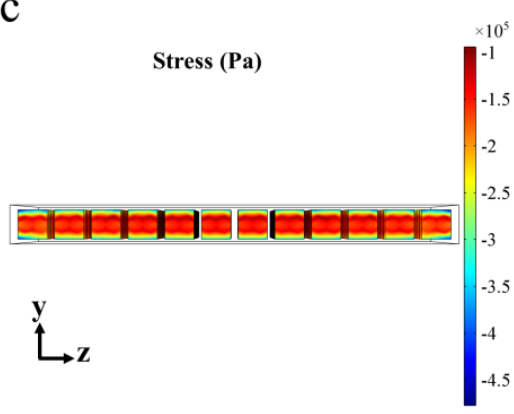

$\mathrm{b}$

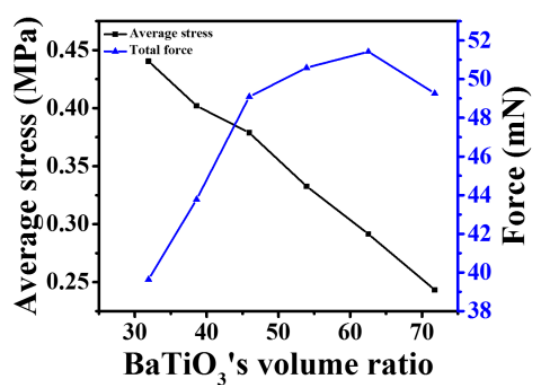

d

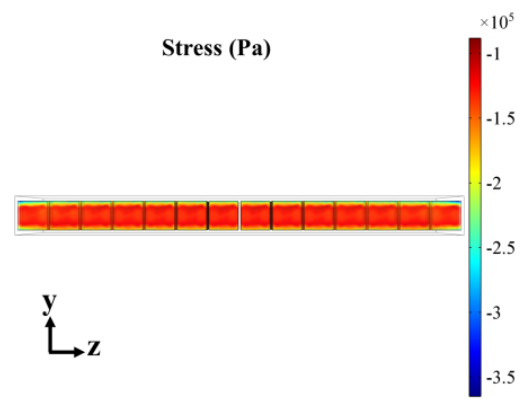

Figure 3. (a) The PENG's top surface charge and capacitance with different $\mathrm{BaTiO}_{3}$ ratios. (b) The averaged stress and total force on the top surface of the embedded $\mathrm{BaTiO}_{3}$ cubes. The distribution of stress in the $\mathrm{BaTiO}_{3}$ at $45.9 \%$ (c) and $62.5 \%(\mathbf{d})$. 
In the experiment, the polymer's Young's modulus and permittivity are not unique as they are often influenced by experimental conditions such as the amount and temperature of curing agent. In order to examine whether the optimal volume ratio changes with these uncertain factors, the open circuit voltage of PENG with $\mathrm{BaTiO}_{3}$ cubes volume ratio around the optimal value is studied. The results are shown in Figure $4 a, b$. When the polymer's permittivity was increased, the voltage decreased notably, which is ascribed to the PENG's increased capacitance. Although the voltage of the PENG is altered when the polymer's Young's modulus or permittivity are changed, the PENG with $\mathrm{BaTiO}_{3}$ volume ratio of $45.9 \%$ still has the max voltage. According to the experimental results [21] published recently, a paper-based PENG composed of $\mathrm{BaTiO}_{3}$ nanoparticles and bacterial cellulose got its maximum voltage when the mass ratio of uniformly distributed $\mathrm{BaTiO}_{3}$ nanoparticles reached $80 \%$. The Young's modulus, relative permittivity, and density of the bacterial cellulose are $10 \mathrm{GPa}, 7.8,1.2 \mathrm{~g} / \mathrm{cm}^{3}$ respectively [24,25]. The PDMS's Young's modulus and relative permittivity are tuned to $10 \mathrm{GPa}$ and 7.8 , the optimal volume ratio of $\mathrm{BaTiO}_{3}$ is still $45.9 \%$ which can be seen in Figure $4 a, b$. In consideration of the density of $\mathrm{BaTiO}_{3}$ and bacterial cellulose at $6.02 \mathrm{~g} / \mathrm{cm}^{3}$ and $1.2 \mathrm{~g} / \mathrm{cm}^{3}$, the optimal mass ratio is $81 \%$. Therefore, our simulation results are in consistent with those experimental results. The distance between the electrode and the $\mathrm{BaTiO}_{3}$ cubes was studied. As shown in Figure 4c, the PENG's voltage increases with the increase of this distance, which may be ascribed to more stress delivered from the PDMS to the $\mathrm{BaTiO}_{3}$ cubes.

a

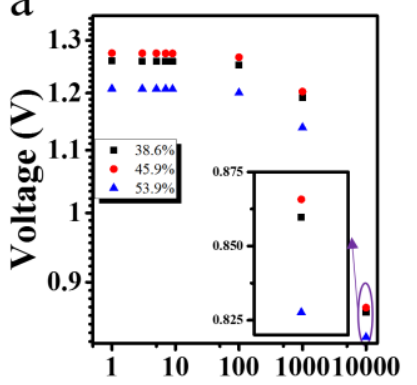

Young's modulus (MPa)
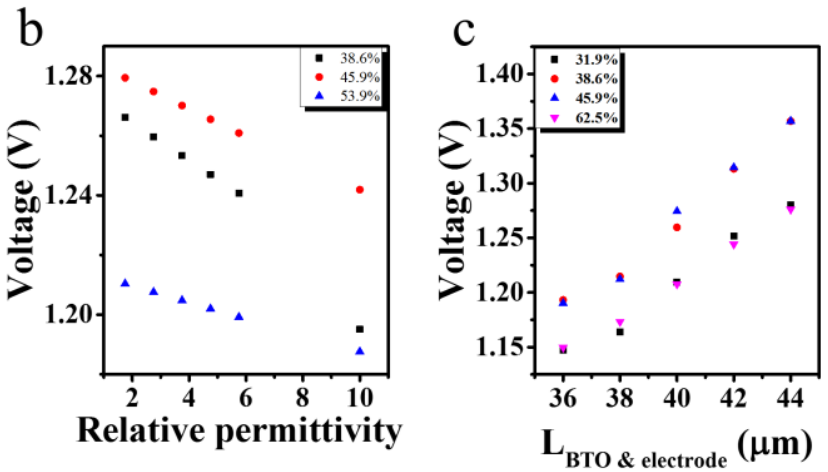

Figure 4. The open circuit voltage of the PENG at different polymer Young's modulus (a), permittivity (b), and the distance between electrode and $\mathrm{BaTiO}_{3}$ cubes (c).

To convert the mechanical energy into electricity, a dynamic force must be exerted on the PENG. So the output of the PENG under the dynamic force was studied. A periodic square wave form force with an amplitude of $0.5 \mathrm{MPa}$ was exerted on the PENG. According to the previous studies, a simple RC circuit with the PENG as the voltage source as shown in Figure 5a can be used to describe the PENG's dynamic electric characters [26], the output of the voltage source can be approximated with a quite high accuracy as [27]

$$
V(t)=d_{33} f(t) / C
$$

where, $d_{33}$ is the piezoelectric constant of the PENG, and $f(t)$ is the dynamic force exerted on the PENG. When the PENG is driven by a harmonic force $f(t) e^{i \omega t}$, where $\omega$ is the angular frequency of the force, the voltage drop across the external load has an analytic expression,

$$
V_{\omega}(t)=\frac{d_{33} f_{\omega}(t) R}{R C+\frac{1}{i \omega}} e^{i \omega t}
$$

Thus, it is convenient to do a spectral analysis of the periodic square wave form force to obtain the voltage drop across the external load. After a spectral analysis of the square wave form force as shown in Figure $5 b$, the PENG's voltage and current output at an external resistance of $50 \mathrm{M} \Omega$ were 
calculated as shown in Figure $5 \mathrm{c}$,d. Under this force, the current and voltage have respective peak values of $1.16 \mathrm{~V}$ and $23.2 \mathrm{nA}$, and the peak power of this PENG is $26.9 \mathrm{nW}$.

a
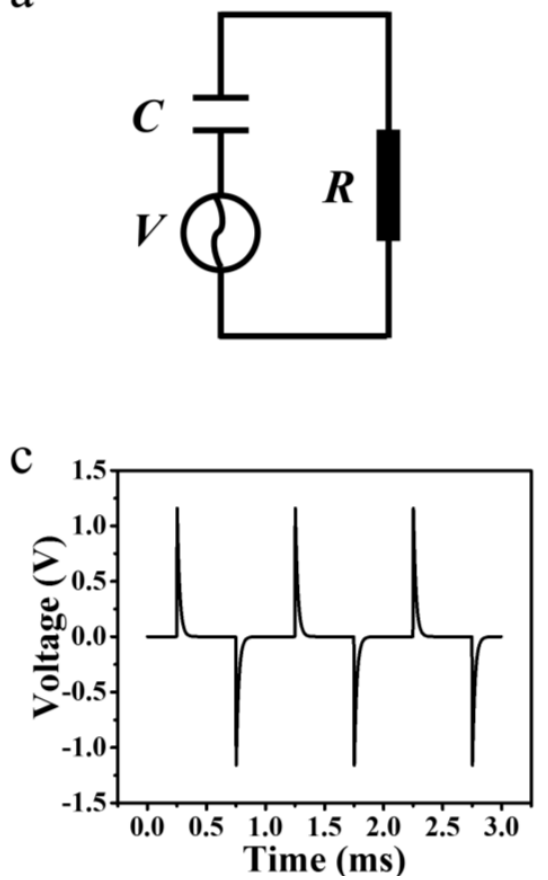

b

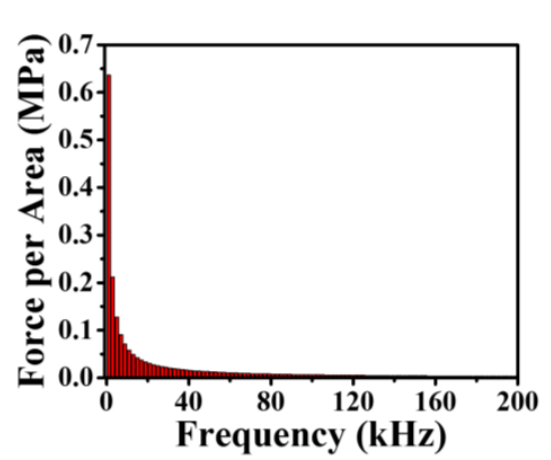

$\mathrm{d}$

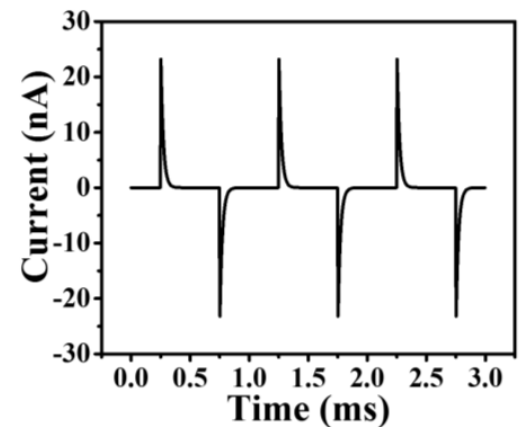

Figure 5. (a) The equivalent circuit of the composite PENG. (b) The spectral analysis of the square wave force. The output voltage (c) and current (d) at an external load with a resistance of $50 \mathrm{M} \Omega$.

\section{Conclusions}

In this paper, we studied the output voltage of the $\mathrm{BaTiO}_{3} / \mathrm{PDMS}$ composite PENG with different volume ratios of evenly distributed $\mathrm{BaTiO}_{3}$ cubes, and find that an optimal volume ratio (46\%) of the $\mathrm{BaTiO}_{3}$ cubes exists at which the PENG can output the highest voltage. The optimal ratio is a result of the trade-off between the surface charge and capacitance of the composite PENG. This optimal volume ratio is stable even if the Young's modulus and permittivity of the polymer matrix are changed. Finally, after a $0.5 \mathrm{MPa}, 1000 \mathrm{~Hz}$ square wave force was exerted, the PENG at the optimal volume ratio can output a current of $23.2 \mathrm{nA}$, voltage of $1.16 \mathrm{~V}$ on the external load with resistance of $50 \mathrm{M} \Omega$.

Acknowledgments: We sincerely appreciate the support from NSFC (No. 51322203, 51472111), the National Program for Support of Top-notch Young Professionals, the Fundamental Research Funds for the Central Universities (No. lzujbky-2016-k02).

Author Contributions: Yong Qin and Qi Xu conceived and designed the calculations; Xi Zhou performed the calculations; Xi Zhou, Qi Xu, Suo Bai, and Yong Qin analyzed the data; Qi Xu wrote the paper. Yong Qin and Weisheng Liu final approval of the version to be published.

Conflicts of Interest: The authors declare no conflict of interest.

\section{References}

1. Wang, Z.L.; Song, J.H. Piezoelectric nanogenerators based on zinc oxide nanowire arrays. Science 2006, 312, 242-246. [CrossRef] [PubMed]

2. Shi, Q.; Wang, T.; Lee, C. MEMS based broadband Piezoelectric Ultrasonic Energy Harvester (PUEH) for enabling self-powered implantable biomedical devices. Sci. Rep. 2016, 6, 24946. [CrossRef] [PubMed]

3. Lu, B.; Chen, Y.; Ou, D.; Chen, H.; Diao, L.; Zhang, W.; Zheng, J.; Ma, W.; Sun, L.; Feng, X. Ultra-flexible piezoelectric devices integrated with heart to harvest the biomechanical energy. Sci. Rep. 2015, 5, 16065. [CrossRef] [PubMed] 
4. Cheng, X.; Xue, X.; Ma, Y.; Han, M.; Zhang, W.; Xu, Z.; Zhang, H.; Zhang, H. Implantable and self-powered blood pressure monitoring based on a piezoelectric thinfilm: Simulated, in vitro and in vivo studies. Nano Energy 2016, 22, 453-460. [CrossRef]

5. Hwang, G.-T.; Park, H.; Lee, J.-H.; Oh, S.; Park, K.-I.; Byun, M.; Park, H.; Ahn, G.; Jeong, C.K.; No, K.; et al. Self-powered cardiac pacemaker enabled by flexible single crystalline PMN-PT piezoelectric energy harvester. Adv. Mater. 2014, 26, 4880-4887. [CrossRef] [PubMed]

6. Yuan, M.; Cheng, L.; Xu, Q.; Wu, W.; Bai, S.; Gu, L.; Wang, Z.; Lu, J.; Li, H.; Qin, Y.; et al. Biocompatible nanogenerators through high piezoelectric coefficient $0.5 \mathrm{Ba}\left(\mathrm{Zr}_{0.2} \mathrm{Ti}_{0.8}\right) \mathrm{O}_{3}-0.5\left(\mathrm{Ba}_{0.7} \mathrm{Ca}_{0.3}\right) \mathrm{TiO}_{3}$ nanowires for in vivo applications. Adv. Mater. 2014, 26, 7432-7437. [CrossRef] [PubMed]

7. Kim, B.-Y.; Lee, W.-H.; Hwang, H.-G.; Kim, D.-H.; Kim, J.-H.; Lee, S.-H.; Nahm, S. Resistive switching memory integrated with nanogenerator for self-powered bioimplantable devices. Adv. Funct. Mater. 2016, 26, 5211-5221. [CrossRef]

8. Siddiqui, S.; Kim, D.-I.; Le Thai, D.; Minh Triet, N.; Muhammad, S.; Yoon, W.-S.; Lee, N.-E. High-performance flexible lead-free nanocomposite piezoelectric nanogenerator for biomechanical energy harvesting and storage. Nano Energy 2015, 15, 177-185. [CrossRef]

9. Siddiqui, S.; Kim, D.-I.; Roh, E.; Duy, L.T.; Trung, T.Q.; Nguyen, M.T.; Lee, N.-E. A durable and stable piezoelectric nanogenerator with nanocomposite nanofibers embedded in an elastomer under high loading for a self-powered sensor system. Nano Energy 2016, 30, 434-442. [CrossRef]

10. Proto, A.; Penhaker, M.; Bibbo, D.; Vala, D.; Conforto, S.; Schmid, M. Measurements of generated energy / electrical quantities from locomotion activities using piezoelectric wearable sensors for body motion energy harvesting. Sensors 2016, 16, 524. [CrossRef] [PubMed]

11. Hu, C.; Cheng, L.; Wang, Z.; Zheng, Y.; Bai, S.; Qin, Y. A transparent antipeep Piezoelectric nanogenerator to harvest tapping energy on screen. Small 2016, 12, 1315-1321. [CrossRef] [PubMed]

12. Lee, S.; Hinchet, R.; Lee, Y.; Yang, Y.; Lin, Z.H.; Ardila, G.; Montes, L.; Mouis, M.; Wang, Z.L. Ultrathin nanogenerators as self-powered/active skin sensors for tracking eye ball motion. Adv. Funct. Mater. 2014, 24, 1163-1168. [CrossRef]

13. Delnavaz, A.; Voix, J. Flexible piezoelectric energy harvesting from jaw movements. Smart Mater. Struct. 2014, 23, 105020. [CrossRef]

14. Liu, X.; Zhao, H.; Lu, Y.; Li, S.; Lin, L.; Du, Y.; Wang, X. In vitro cardiomyocyte-driven biogenerator based on aligned piezoelectric nanofibers. Nanoscale 2016, 8, 7278-7286. [CrossRef] [PubMed]

15. Lin, Y.; Deng, P.; Nie, Y.; Hu, Y.; Xing, L.; Zhang, Y.; Xue, X. Room-temperature self-powered ethanol sensing of a Pd/ZnO nanoarray nanogenerator driven by human finger movement. Nanoscale 2014, 6, 4604-4610. [CrossRef] [PubMed]

16. Park, K.-I.; Lee, M.; Liu, Y.; Moon, S.; Hwang, G.-T.; Zhu, G.; Kim, J.E.; Kim, S.O.; Kim, D.K.; Wang, Z.L.; et al. Flexible nanocomposite generator made of $\mathrm{BaTiO}_{3}$ nanoparticles and graphitic carbons. Adv. Mater. 2012, 24, 2999-3004. [CrossRef] [PubMed]

17. Park, K.-I.; Jeong, C.K.; Ryu, J.; Hwang, G.-T.; Lee, K.J. Flexible and large-area nanocomposite generators based on lead zirconate titanate particles and carbon nanotubes. Adv. Energy Mater. 2013, 3, 1539-1544. [CrossRef]

18. Jeong, C.K.; Park, K.-I.; Ryu, J.; Hwang, G.-T.; Lee, K.J. Large-Area and flexible lead-free nanocomposite generator using alkaline niobate particles and metal nanorod filler. Adv. Funct. Mater. 2014, 24, 2620-2629. [CrossRef]

19. Sultana, A.; Alam, M.M.; Garain, S.; Sinha, T.K.; Middya, T.R.; Mandal, D. An effective electrical throughput from PANI supplement ZnS nanorods and PDMS-based flexible piezoelectric nanogenerator for power up portable electronic devices: An alternative of MWCNT filler. ACS Appl. Mater. Interfaces 2015, 7, 19091-19097. [CrossRef] [PubMed]

20. Jeong, C.K.; Kim, I.; Park, K.-I.; Oh, M.H.; Paik, H.; Hwang, G.-T.; No, K.; Nam, Y.S.; Lee, K.J. Virus-directed design of a flexible $\mathrm{BaTiO}_{3}$ nanogenerator. ACS Nano 2013, 7, 11016-11025. [CrossRef] [PubMed]

21. Zhang, G.; Liao, Q.; Zhang, Z.; Liang, Q.; Zhao, Y.; Zheng, X.; Zhang, Y. Novel piezoelectric paper-based flexible nanogenerators composed of $\mathrm{BaTiO}_{3}$ nanoparticles and bacterial cellulose. Adv. Sci. 2016, 3, 1500257. [CrossRef] [PubMed] 
22. Lee, K.Y.; Kim, D.; Lee, J.-H.; Kim, T.Y.; Gupta, M.K.; Kim, S.-W. Unidirectional high-power generation via stress-induced dipole alignment from $\mathrm{ZnSnO}_{3}$ nanocubes/polymer hybrid piezoelectric nanogenerator. Adv. Funct. Mater. 2014, 24, 37-43. [CrossRef]

23. Gao, Y.; Wang, Z.L. Electrostatic potential in a bent piezoelectric nanowire. The fundamental theory of nanogenerator and nanopiezotronics. Nano Lett. 2007, 7, 2499-2505. [CrossRef] [PubMed]

24. Andrianov, A.V.; Aleshin, A.N.; Khripunov, A.K.; Trukhin, V.N. Terahertz properties of bacterial cellulose films and its composite with conducting polymer PEDOT/PSS. Synth. Met. 2015, 205, 201-205. [CrossRef]

25. Henriksson, M.; Berglund, L.A.; Isaksson, P.; Lindstrom, T.; Nishino, T. Cellulose nanopaper structures of high toughness. Biomacromolecules 2008, 9, 1579-1585. [CrossRef] [PubMed]

26. Zhou, Y.; Liu, W.; Huang, X.; Zhang, A.; Zhang, Y.; Wang, Z.L. Theoretical study on two-dimensional MoS 2 piezoelectric nanogenerators. Nano Res. 2016, 9, 800-807. [CrossRef]

27. Xu, Q.; Qin, Y. Theoretical study of enhancing the piezoelectric nanogenerator's output power by optimizing the external force's shape. APL Mater. 2017, 5, 074101. [CrossRef]

(C) 2017 by the authors. Licensee MDPI, Basel, Switzerland. This article is an open access article distributed under the terms and conditions of the Creative Commons Attribution (CC BY) license (http:// creativecommons.org/licenses/by/4.0/). 